\title{
Thrombin-activated platelet rich plasma (PRP) enhanced osteogenic differentiation of bone marrow mesenchymal stem cells by activing autophagy through miR-140-3p/SPRED2 axis
}

Shuting Jiang

Henan Provincial People's Hospital

Hongyan Liu

Henan Provincial People's Hospital

Weiyan Zhu

Henan Provincial People's Hospital

Hui Yan

Henan Provincial People's Hospital

Beizhan Yan ( $\sim$ Beizhan_yan5544@163.com )

Henan Provincial People's Hospital

\section{Research}

Keywords: PRP, BMSCs, osteogenic differentiation, autophagy, miR-140-3p, SPRED2

Posted Date: December 31st, 2020

DOI: https://doi.org/10.21203/rs.3.rs-136527/v1

License: (c) (i) This work is licensed under a Creative Commons Attribution 4.0 International License.

Read Full License 


\section{Abstract}

Background Mesenchymal stem cells transplantation gradually become a potential treatment for bone defect in clinic practice. This study aimed to investigate the molecular mechanism of PRP and autophagy for osteogenic differentiation in bone marrow mesenchymal stem cells (BMSCs).

Methods Thrombin activated PRP was prepared and the BMSCs were treated with activated PRP with different concentration and transfected with miR-140-3p vector (mimics or inhibitor), si-SPRED2 or cotransfected with miR-140-3p inhibitor and si-SPRED2, respectively. qRT-PCR and Western blotting were used to determine the mRNA expression and protein expression. A luciferase reporter assay was conducted to identified the targeting relationship between iR-140-3p and SPRED2 Subsequently, cell proliferation was detected by MTT and ALP activity was also determined. Alizarin red staining was used for the evaluating the formation of calcium nodules.

Results MiR-140-3p expression was found to be inhibited by PRP in a dose-dependent manner, besides, cell proliferation, ALP activity, the expression of COL-I, OPN, Runx2 and OCN, and the formation of calcium nodules related to osteogenic differentiation were enhanced by PRP. Subsequently, we found that PRP activated autophagy and up-regulated SPRED2 expression in BMSCs through suppressing miR-140$3 p$ expression. Moreover, we confirmed that miR-140-3p targeted SPRED2 and negatively regulation its expression. Finally, the findings showed that inhibition of miR-140-3p enhanced cell proliferation, osteogenic differentiation and autophagy of BMSCs by negatively regulating SPRED2 expression.

Conclusion Thrombin activated PRP accelerated osteogenic differentiation of BMSCs by activing autophagy through miR-140-3p/SPRED2 axis.

\section{Introduction}

In order to enhance bone regeneration, various approaches, including bone transport and distraction osteogenesis, have been conducted for the repair of bone defect in the last decades [1]. However, these therapies might lead to donor site injury, infection, deformity, surgical risks and even healthy bone loss [2]. Therefore, mesenchymal stem cells transplantation gradually become a potential treatment for bone defect in clinic practice.

Numerous studies revealed that bone marrow mesenchymal stem cells (BMSCs) have the potential to differentiate into osteoblasts, myoblasts, adipocytes and so on under different conditions, representing an alternative reparative cell type [3]. And the osteogenesis of BMSCs is always considered as a potential therapeutic strategy for bone regeneration. However, the molecular mechanisms for differential of BMSCs are still unclear.

In addition to stem cell therapy, platelet rich plasma (PRP) has also been widely applied in various fields, especially bone detects in maxillofacial region and oral cavity [4]. Moreover, PRP at different concentrations showed different potentials to induce chondrogenic differentiation, migration, and 
proliferation of mesenchymal progenitor cells [5]. Recently, more and more researches show that PRP can be used in the field of bone regeneration, and it has been involved widely in orthopedic surgery [6]. It was found PRP could be used in treatment of chronic lateral elbow epicondylitis, mandibular degree II furcation defects and tendinopathy [7-9]. And the application of PRP is considered as a potential therapeutic strategy in bone repair in the future[10]. However, the underlying molecular mechanisms for PRP in osteogenic differentiation are still unknown. Interesting, PRP was reported to activate autophagy in osteoblast precursor 3T3-L1 [11]. Thus, we wondered the relationship between PRP and autophagy in osteogenic differentiation in BMSCs.

In the present study, we investigated the molecular mechanism of PRP and autophagy in BMSCs. We for the first time demonstrated that thrombin-activated PRP enhanced osteogenic differentiation of BMSCs by activing autophagy through miR-140-3p/SPRED2 axis. This study might provide more research basis and might give novel study targets for PRP in clinical treatment of bone regeneration.

\section{Materials And Methods}

\section{Preparation of Thrombin Activated Platelet-Rich Plasma(PRP)}

For the preparation of PRP, human blood was collected from 8 donors ( $150 \mathrm{~mL}$ of venous blood each) and mixed with $10 \%$ acid-citrate-dextrose as an anticoagulant. The whole blood was centrifuged for 25 min and PRP was extracted from the buffycoat into an empty sterile syringe. For the activation, bovine thrombin was added to PRP at 1:5(vol/vol) and incubated for 30 minutes at $37^{\circ} \mathrm{C}$. Then the sample was centrifuged at $4000 \mathrm{rpm}$ for $5 \mathrm{~min}$, and the supernatant was collected and mixed with serum-free DMEM solution to prepare PRP at a concentration of $5 \%, 10 \%$ and $20 \%$.

\section{Cell Culture and Transfection}

The human bone marrow stem cells (BMSCs) were obtained from ATCC (Rockville, MD, USA) and cultured in Dulbecco's modified Eagle Medium (DMEM) including 10\% fetal bovine serum (FBS), $100 \mathrm{IU} / \mathrm{ml}$ penicillin and $100 \mu \mathrm{g} / \mathrm{ml}$ streptomycin. Cells were incubated in a humidified atmosphere at $37^{\circ} \mathrm{C}$ with $5 \%$ $\mathrm{CO}_{2}$. BMSCs were treated with activated PRP at different concentration and transfected with miR-140-3p vector (mimics or inhibitor), si-SPRED2 or co-transfected with miR-140-3p inhibitor and si-SPRED2, respectively.

\section{MTT Assay}

The cell viability was detected by MTT assay at $0 \mathrm{~h}, 24 \mathrm{~h}, 48 \mathrm{~h}, 72 \mathrm{~h}$ and $96 \mathrm{~h}$ after the treatment. BMSCs were planted into 96 -well microplates with the density of $5 \times 10^{3}$ and incubated for $48 \mathrm{~h}$. subsequently, 10 $\mu \mathrm{L}$ of $5 \mathrm{mg} / \mathrm{mL}$ MTT solution was added to each well and incubated for another $4 \mathrm{~h}$ at $37^{\circ} \mathrm{C}$. The supernatant was discarded and replaced with $150 \mu \mathrm{l}$ DMSO. Finally, the absorbance was measured was measured at $490 \mathrm{~nm}$ by using a microplate reader (DNM-9602; Pe rlong, Beijing, China). Each experiment was performed in triplicate and repeated at least three times.

\section{Alizarin Red Staining and Alkaline Phosphatase (ALP) Activity}


BMSCs were fixed with $4 \%$ paraformaldehyde and incubated with $1 \%$ alizarin red S solution (Beyotime, Shanghai, China) for 10 min at room temperature. Excess stain was eliminated using PBS washing. Calcium-bound stain was collected with $100 \mathrm{mM} \mathrm{CPC}$ and absorbance value was measured at $405 \mathrm{~nm}$ by a microplate reader.

For the detection of ALP activity, BMSCs were cultured in 24-well plates at a cell density of $1 \times 10^{4}$ for 7 days, washed with PBS and fixed with $4 \%$ paraformaldehyde. Protein levels were determined by a BCA protein assay kit and the ALP activity was measured by using a ALP detection kit (Wako Chemical, Richmond, VA).

\section{Reverse Transcription-Quantitative Polymerase Chain Reaction (qRT-PCR)}

In brief, Total RNA was extracted from BMSCs using TRIzol reagent (Invitrogen; Thermo Fisher Scientific, Inc.). For the detection of miRNA expression, RNA was firstly converted into cDNA by using a iScriptTM cDNA Synthesis Kit (Bio-Rad, Reinach, Switzerland). A SYBR Green PCR Master Mix (Applied Biosystems, Foster City, CA, USA) on a ABI Prism 7300 (Applied Biosystems) was used for quantification. For the detection of mRNA expression, DyNamoTM cDNA synthesis kit (Fermentas) was used for the reverse transcription. PCR reaction and analysis was performed on an ABI Prism 7500 sequence detection system (PE Applied Biosystems) with a DyNamo SYBR1 Green qPCR kit (Finnzymes, Espoo, Finland). U6 and GAPDH were used as internal controls for miRNA and $m R N A$, respectively. The following primers were used in PCRs: F 5'-GCTTGGTGGGCTTCTGGT-3' and R 5'-CCCGGTATCCTGTCCTGTCCGTGGT-3' for miR-140-3p; F 5'-CTCATCCATGGTGAACGACAGAA-3' and R 5'-TGTCAAAGGCTCGGGCATC-3' for SPRED2; F 5'-GAGAGCATGACCGATGGAT-3' and R 5'-ATGTTTTGGTGGTTCAGGAGG-3' for COL-1; F 5'GTGCAGCCTHGTGTCCAAG-3' and R 5'-GTCAGCCAACTCGTCACAGT-3' for OCN; F 5'CTGAACGCGCCTTCTGATTG-3' and R 5'-ACATCGGAATGCTCATTGCTCT-3' for OPN; F 5'GGAGTGGACGAGGCAAGAGTTT-3' and 5'-AGCTTCTGTCTGTGCCTTCTGG-3' for Runx2; F 5'TGCGGGTGCTCGCTTCGGCAGC-3' and R 5'-CCAGTGCAGGGTCCGAGGT-3' for U6; F 5'ACAGAGCCTCGCCTTTGC-3' and R 5'-GAGGCGTACAGGGATAGCAC-3' for $\beta$-actin. The relative expression levels of miRNA or mRNA was calculated by the $2^{-\triangle \triangle C q}$ method.

\section{Western Blotting}

Protein was extracted by RIPA lysis buffer (Thermo Scientific, U.S.A.) from BMSCs and determined using a BCA Protein Assay Kit (ComWin Biotech, Beijing, China). Equal amount of proteins was loaded on 10\% SDS-PAGE gels, transferred to PVDF membranes and blocked with $5 \%$ non-fat milk at room temperature for $1 \mathrm{~h}$. Afterwards, membranes were subjected to immunoblotting with primary antibodies at $4^{\circ} \mathrm{C}$ overnight, followed by horseradish peroxidase-conjugated secondary antibodies at $37^{\circ} \mathrm{C}$ for $1 \mathrm{~h}$. The primary antibodies were listed as follows: SPRED2(ab153700, 1/500)), Beclin1 (ab207612, 1/2000) and LC3 I/II (ab62721, 1/1000) (all from Abcam, Cambridge, MA, U.S.A). $\beta$-actin was used as a control. Immunoreactive bands were visualized using an enhanced chemiluminescence detection system (Amersham Biosciences, Piscataway, NJ, USA) and quantified using AlphaEase FC software.

\section{Luciferase Reporter Assay}


Two types of 3'-UTRs (WT and MUT) of SPRED2 sequences containing the binding sequence of miR-140$3 p$ were amplified and inserted into the p-MIR-report plasmid (Ambion, Austin, TX, USA). Subsequently, BMSCs were transfected with miR-140-3p vector (mimics or inhibitor) and SPRED2 luciferase reporter plasmid (WT- SPRED2 or MUT- SPRED2) or NC (mimics or inhibitor) using Lipofectamine 2000 (GeneChem, Shanghai, China). The luciferase activity was detected using luciferase assay kits (Promega, Madison, Wisconsin, U.S.A.) after the transfection of $48 \mathrm{~h}$. Luciferase activity of cells was normalized to Renilla luciferase activity.

\section{Statistical Analysis}

All calculations were performed using SPSS 18.0. The continuous data was expressed as mean \pm SD. Comparison between two groups was made using the Student t-test, while, comparison among three or more groups was perfomed using one-way analysis of variance (ANOVA) following by Turkey analysis. The comparison for rates was made by Chi square test. The differences were considered statistically significant when $\mathrm{P}<0.05$.

\section{Results}

PRP promotes the proliferation and osteogenic differentiation of human BMSCs by miR-140-3p inhibition or autophagy activation

BMSCs were transfected miR-140-3p mimics and NC mimics, respectively. QRT-PCR result confirm miR140-3p was dramatically up-regulated by the transfection with miR-140-3p mimics (Fig. 1A). Subsequently, cells were treated with thrombin-activated PRP at a concentration of $5 \%, 10 \%$ and $20 \%$, respectively. MiR-140-3p was down-regulated in cells treated with PRP in a dose-dependent manner (Fig. 1B). Cell proliferation detected by MTT assay showed that thrombin-activated PRP notably enhanced cell viability in a dose-dependent manner but up-regulated miR-140-3p or inhibited autophagy reversed the effect of activated PRP on cell proliferation (Fig. 1C). ALP activity was also found to be increased by activated PRP in a dose-dependent manner that was reversed by miR-140-3p mimics or 3MA (Fig. 1D). Besides, osteogenic differentiation related mRNA expression of COL-I, OPN, Runx2 and OCN was significantly elevated by activated PRP in a dose-dependent manner but down-regulated by overexpressed miR-140-3p or inhibited autophagy (Fig. 1E). Alizarin red staining illustrated that thrombinactivated PRP obviously accelerated formation of calcium nodules in a dose-dependent manner, which could be reversed by overexpressed miR-140-3p or inhibited autophagy (Fig. 1F). All the results indicated that PRP promotes cell proliferation and osteogenic differentiation of human BMSCs by miR-140-3p inhibition or autophagy activation.

\section{PRP activates autophagy and up-regulated SPRED2 expression of human BMSCs through suppressing miR-140-3p expression}

We further investigated the regulation of miR-140-3p on autophagy and SPRED2 expression in BMSCs treated with thrombin-activated PRP. As shown in Fig. 2A, the expression of SPRED2 was up-regulated by the treatment of PRP, however, it was reversed by overexpressed miR-140-3p or autophagy inhibition. 
Western blotting result also showed that the protein level of Beclin1 and SPRED2, and the conversion rate of LC3 I to LC3 II was significantly elevated by PRP in a dose-manner but reversed by miR-140-3p mimics or autophagy inhibitor (Fig. 2B). The above findings suggested that PRP enhanced autophagy and upregulated SPRED2 expression of human BMSCs through suppressing miR-140-3p expression.

\section{MiR-140-3p negatively regulated SPRED2 expression}

Next, we explored the regulation relationship between miR-140-3p and SPRED2. Based on bioinformatics analysis, miR-1 40-3p could directly bind to the SPRED2 3'-UTR (Fig. 3A). Luciferase reporter assay revealed that fluorescence intensity of WT-SPRED2 was significantly decreased by miR-140-3p mimics but increased by miR-140-3p inhibitor, however, fluorescence intensity of MUT-SPRED2 was affected by neither of them (Fig. 3B). QRT-PCR and western blotting detection also confirmed that SPRED2 expression was obviously suppressed by miR-140-3p mimics but enhanced by miR-140-3p inhibitor (Fig. 3C-D). these results demonstrated that miR-140-3p targeted SPRED2 and negatively regulation its expression.

\section{Inhibition of miR-140-3p enhances cell proliferation and osteogenic differentiation of BMSCs by negatively regulating SPRED2 expression}

We further studied the molecular mechanism of miR-140-3p for cell proliferation and osteogenic differentiation of BMSCs. Firstly, cells were transfected with si-SPRED2 or miR-140-3p inhibitor, respectively. Both qRT-PCR and western blotting result showed that SPRED2 and miR-140-3p was knockdown successful (Fig. 4A). As shown in Fig. 4B, cell proliferation was suppressed by SPRED2 knockdown. And miR-140-3p inhibitor obviously increased cell proliferation, which was reversed by the transfection of si-SPRED2. ALP activity (Fig. 4C) was also reduced by down-regulated SPRED2. It was also found that miR-140-3p knockdown attenuated ALP activity which could be reversed by si-SPRED2. Moreover, protein level of COL-I, OPN, Runx2, OCN was decreased by si-SPRED2 compared to NC control in cells treated with $20 \%$ PRP (Fig. 4D). Down-regulated miR-140-3p was also proven to enhance the expression of above proteins related to osteogenic differentiation that could be reversed by si-SPRED2. In addition, knockdown of si-SPRED2 suppressed calcium deposition, and miR-140-3p inhibitor accelerated the formation of calcium nodules but it was reversed by si-SPRED2 (Fig. 4E). these finding illustrated that inhibition of miR-140-3p enhanced cell proliferation and osteogenic differentiation of BMSCs by negatively regulating SPRED2 expression.

\section{Knockdown of miR-140-3p enhances autophagy in human BMSCs through negatively regulating SPRED2 expression}

Finally, the molecular mechanism of miR-140-3p on autophagy in BMSCs was studied. As shown in Fig. 5A, the expression of SPRED2 mRNA was decreased in cells treated with $20 \%$ PRP but up-regulated in cells transfected with miR-140-3p inhibitor, which was reversed by the co-transfection of si-SPRED2. Furthermore, western blotting revealed that the expression of SPRED2 and beclin1 as well as the conversion rate of LC3 I to LC3 II was notably decreased in cells treated with PRP, however, the levels of 
them were elevated in cells transfected with miR-140-3p inhibitor but was decreased by the cotransfection of si-SPRED2. These findings indicated that miR-140-3p suppression enhanced autophagy in BMSCs through negatively regulating SPRED2 expression.

\section{Discussion}

BMSCs are a type of primary stem cells with good differentiation potential, widely using in tissue engineering for translational research and clinical applications [12]. In recent years, several studies reported the roles of various proteins and miRNAs in the treatment of bone defect [13, 14], however, the mechanism of miRNA-140-3p, SPRED2 and thrombin in BMSCs as well the target relationship still remains unclear. In the present study, we demonstrated that thrombin-activated platelet rich plasma (PRP) enhanced osteogenic differentiation of bone marrow mesenchymal stem cells through miR-1403p/SPRED2-mediated autophagy.

MiRNAs are reported to be involved in in a variety of biological processes, including cell viability, apoptosis and differentiation[15]. MiR-140-3p was reported to regulated osteogenic differentiation of different cells in vivo and in vitro studie, besides, overexpressed miR-140-3p suppressed the cell viability and differentiation, and enhanced the apoptosis of preosteoblasts cell MC3T3-E1 [16]. It was also found that miR-140-5p restrained osteogenesis mediated by BMP2 in human mesenchymal stem cells [17]. Yin et al found that miR-140-3p was up-regulated in peripheral blood mononuclear of patients with osteoporosis patients, besides, the in vitro study showed that miR-140-3p regulated the cell proliferation and differentiation of osteoblasts and osteoclasts by targeting PTEN [18]. Another study found that miR140-3p was up-regulated in patients with osteoporosis in postmenopausal women and it was considered as a potential biomarker [19]. Though PRP was reported to accelerate osteogenic differentiation in mesenchymal stem cells [20], dental stem cells [21], adipose-derived stem cells [22] and so on, the effect of PRP on miR-140-3p was not investigated and no research illustrating the regulation of miR-140-3p for autophagy in BMSCs. In the present study, we found that activated PRP accelerated osteogenic differentiation, cell proliferation and autophagy by inhibiting miR-140-3p expression. However, there is no research illustrating the regulation of miR-140-3p for autophagy in BMSCs.

Circulating evidences showed that Sprouty-related EVH1 domain-2(SPRED2) was potent inhibitors for cytokines and growth factors [23]. SPRED2 was reported to be involved in various diseases [24]. For example, down-regulated SPRED2 induced cardiac arrhythmias and premature death by suppressing autophagy [25]. SPRED2 interacted with LC3 and enhanced autophagy in tumor cells [26]. In addition, SPRED2 was found to induce erythroid differentiation partly through ERK signaling in chronic myeloid leukemia cells [27]. A previous research showed that miR-221-3p could target SPRED2 and SPRED2 regulated LPS-induced lung inflammation via ERK1/2 pathway [28]. However, no studied reported the regulation relationship between SPRED2 and miR-140-3p in BMSCs. Our study for the first time founder that miR-140-3p negatively regulated SPRED2 expression. Besides, down-regulated miR-140-3p enhanced cell proliferation, osteogenic differentiation and autophagy of BMSCs by negatively regulating SPRED2. 


\section{Conclusion}

In summary, we for the first time demonstrated that active PRP accelerate osteogenic differentiation of BMSCs by activing autophagy through miR-140-3p/SPRED2 axis. Our findings might bring a new novel for the target therapy for PRP in bone defect repair in future clinic practice.

\section{Abbreviations}

BMSCs: bone marrow mesenchymal stem cells; DMEM: Dulbecco's medium Eagle's media;DMSO: Dimethylsulfoxide; FBS: Foetal bovine serum; PBS: Phosphate-buffered saline; qRT-PCR: Reverse Transcription-Quantitative Polymerase Chain Reaction; PRP: platelet rich plasma;SPRED2: Sprouty-related EVH1 domain-2; ATCC: American Type Culture Collection; ALP: Alkaline phosphatase; mRNA: messenger RNA; miR: MicroRNA; 3'UTR:3'-Untranslated region; WB: Western blotting

\section{Declarations}

\section{Ethics approval and consent to participate}

The cells were commercially sourced, and the carried work was approved by the Ethics Committee on Henan Provincial People's Hospital.

\section{Consent for publication}

Not applicable

\section{Competing interests}

The authors declare no conflict of interest.

\section{Data Availability}

All data can be obtained from the manuscript or from request to the author.

\section{Funding}

None

\section{Authors' contributions}

Shuting Jiang wrote the original manuscript and conducted the experiments; Hongyan Liu design the study and reviewed the draft; Weiyan Zhu collected the data and conducted analysis; Hui Yan performed statistical analysis and reviewed the manuscript; Beizhan Yan collected data and reviewed manuscript. 
None

\section{References}

1. Elgali, I., et al., Guided bone regeneration: materials and biological mechanisms revisited. European journal of oral sciences, 2017. 125(5): p. 315-337.

2. Yan, Y., et al., Vascularized 3D printed scaffolds for promoting bone regeneration. Biomaterials, 2019. 190: p. 97-110.

3. Chen, $\mathrm{X}$., et al., RANKL signaling in bone marrow mesenchymal stem cells negatively regulates osteoblastic bone formation. Bone research, 2018. 6(1): p. 1-8.

4. Qian, Y., et al., Platelet-rich plasma derived growth factors contribute to stem cell differentiation in musculoskeletal regeneration. Frontiers in chemistry, 2017. 5: p. 89.

5. Kreuz, P.C., et al., Platelet-Rich Plasma Preparation Types Show Impact on Chondrogenic Differentiation, Migration, and Proliferation of Human Subchondral Mesenchymal Progenitor Cells. Arthroscopy: The Journal of Arthroscopic \& Related Surgery, 2015.

6. Fernandes, G. and S. Yang, Application of platelet-rich plasma with stem cells in bone and periodontal tissue engineering. Bone Research, 2016. 4(1): p. 1-21.

7. Thanasas, C., et al., Platelet-rich plasma versus autologous whole blood for the treatment of chronic lateral elbow epicondylitis: a randomized controlled clinical trial. The American journal of sports medicine, 2011. 39(10): p. 2130-2134.

8. Foster, T.E., et al., Platelet-rich plasma: from basic science to clinical applications. The American journal of sports medicine, 2009. 37(11): p. 2259-2272.

9. Fitzpatrick, J., M. Bulsara, and M.H. Zheng, The effectiveness of platelet-rich plasma in the treatment of tendinopathy: a meta-analysis of randomized controlled clinical trials. The American Journal of Sports Medicine, 2017. 45(1): p. 226-233.

10. Dhillon, R.S., E.M. Schwarz, and M.D. Maloney, Platelet-rich plasma therapy-future or trend? Arthritis research \& therapy, 2012. 14(4): p. 219.

11. Carminati, S.A., M.C. Barbosa, and C.M. Fader Kaiser, Platelet rich plasma (PRP) induces autophagy in osteoblast precursor 3T3-L1. 2018.

12. Li, X., et al., Exosomes derived from maxillary BMSCs enhanced the osteogenesis in iliac BMSCs. Oral Diseases, 2020. 26(1): p. 131-144.

13. Li, Y., et al., miR-149-3p regulates the switch between adipogenic and osteogenic differentiation of BMSCs by targeting FTO. Molecular Therapy-Nucleic Acids, 2019. 17: p. 590-600.

14. Dai, Z., et al., MiR-217 promotes cell proliferation and osteogenic differentiation of BMSCs by targeting DKK1 in steroid-associated osteonecrosis. Biomedicine \& Pharmacotherapy, 2019. 109: p. 1112-1119.

15. Zhu, Y.L., et al., miR-217 inhibits osteogenic differentiation of rat bone marrow-derived mesenchymal stem cells by binding to Runx2. Molecular Medicine Reports, 2017. 15(5): p. 3271-3277. 
16. Mao, J.-H., et al., miR-140-3p exhibits repressive functions on preosteoblast viability and differentiation by downregulating MCF2L in osteoporosis. In Vitro Cellular \& Developmental BiologyAnimal, 2020. 56(1): p. 49-58.

17. Hwang, S., et al., miR-140-5p suppresses BMP2-mediated osteogenesis in undifferentiated human mesenchymal stem cells. Febs Letters, 2016. 588(17): p. 2957-2963.

18. Yin, R., et al., miR-140-3p aggregates osteoporosis by targeting PTEN and activating PTEN/PI3K/AKT signaling pathway. Human Cell, 2020. 33(3): p. 569-581.

19. G., R.-S.E., et al., Serum miRNAs miR-140-3p and miR-23b-3p as potential biomarkers for osteoporosis and osteoporotic fracture in postmenopausal Mexican-Mestizo women. Gene, 2018. 679: p. $19-27$.

20. Lin, S.S., et al. Controlled release of PRP-derived growth factors promotes osteogenic differentiation of human mesenchymal stem cells. in International Conference of the IEEE Engineering in Medicine \& Biology Society. 2006.

21. Lee, J.Y., et al., The effects of platelet-rich plasma derived from human umbilical cord blood on the osteogenic differentiation of human dental stem cells. Vitro Cell Dev Biol Anim, 2011. 47(2): p. 157164.

22. Xu, F.T., et al., Effect of activated autologous platelet-rich plasma on proliferation and osteogenic differentiation of human adipose-derived stem cells in vitro. American Journal of Translational Research, 2015. 7(2): p. 257-70.

23. Ohkura, T., et al., Spred2 Regulates High Fat Diet-Induced Adipose Tissue Inflammation, and Metabolic Abnormalities in Mice. Frontiers in immunology, 2019. 10: p. 17.

24. Yang, X., et al., Spred2 deficiency exacerbates D-galactosamine/lipopolysaccharide-induced acute liver injury in mice via increased production of TNFa. Scientific reports, 2018. 8(1): p. 1-12.

25. Ullrich, M., et al., SPRED2 deficiency elicits cardiac arrhythmias and premature death via impaired autophagy. Journal of Molecular and Cellular Cardiology, 2019.

26. Jiang, K., et al., Tumor suppressor Spred 2 interaction with LC3 promotes autophagosome maturation and induces autophagy-dependent cell death. Oncotarget, 2016. 7(18): p. 25652-25667.

27. Yang, Y., et al., Spred2 Modulates the Erythroid Differentiation Induced by Imatinib in Chronic Myeloid Leukemia Cells. PLOS ONE, 2015.

28. Xu, Y., et al., Spred-2 Deficiency Exacerbates Lipopolysaccharide-Induced Acute Lung Inflammation in Mice. PLoS ONE, 2014.

\section{Figures}


A

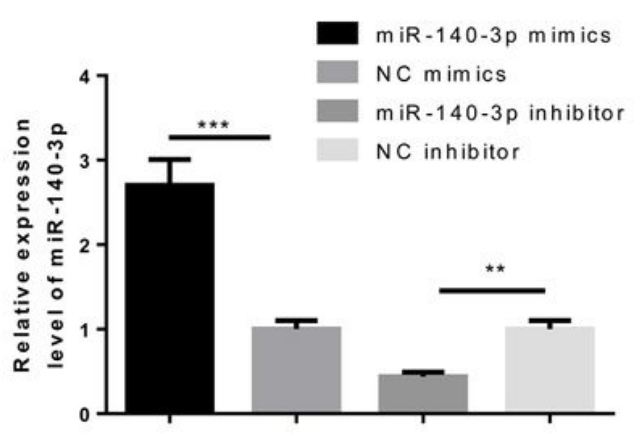

C

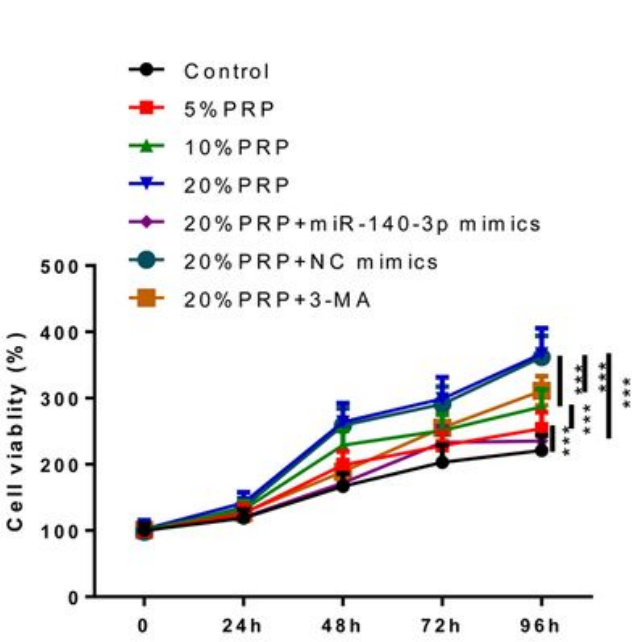

F

\begin{abstract}
D
\end{abstract}

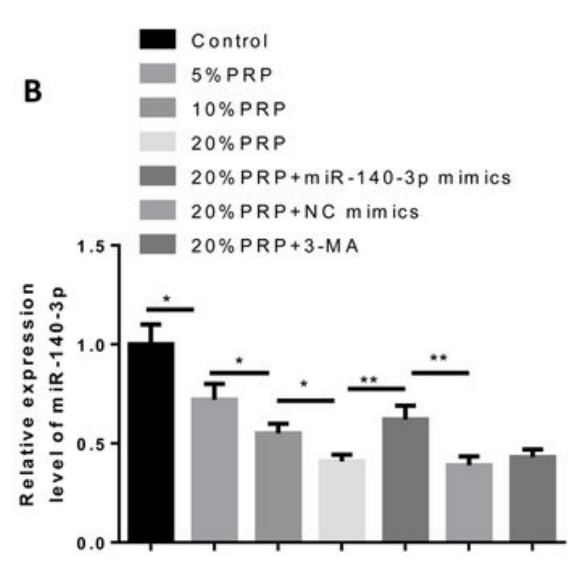

E
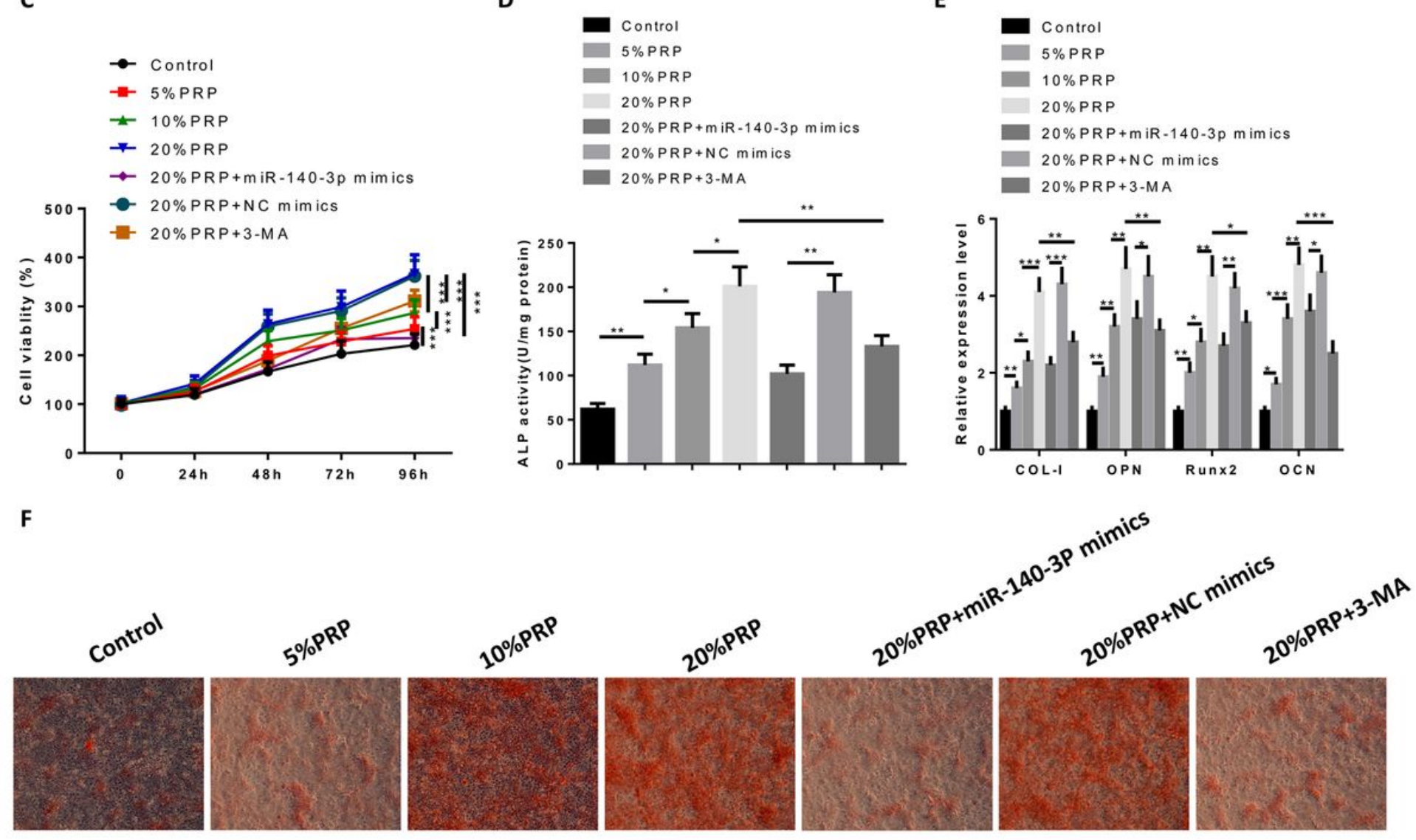

\section{Figure 1}

PRP promotes the proliferation and osteogenic differentiation of human BMSCs by miR-140-3p inhibition or autophagy activation. (A) The transfection efficiency was detected by qRT-PCR. (B) The expression of miR-140-3p was determined by qRT-PCR in BMSCs treated with different concentrations of PRP, BMSCs treated with 20\% PRP and transfected with miR-140-3p mimics, BMSCs treated with 20\% PRP and transfected with NC mimics, and BMSCs treated with 20\% PRP and 3-MA. (C) Cell proliferation was examined by MTT assay. (D) ALP activity was detected. (E) Expression of COL-1, OPN, Runx2 and OCN was measured by qRT-PCR. (F) Calcium deposition was evaluated by Alizarin red staining assay. ${ }^{*} \mathrm{P}<0.05$, $\star * P<0.01, * \star * P<0.001$. 
A

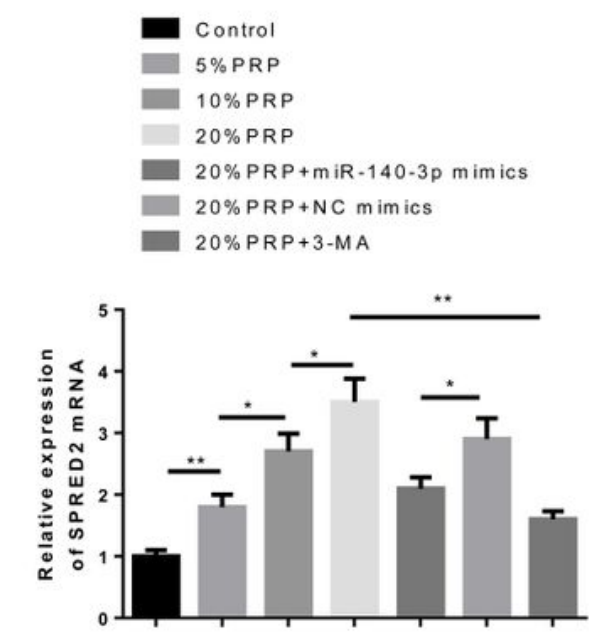

B

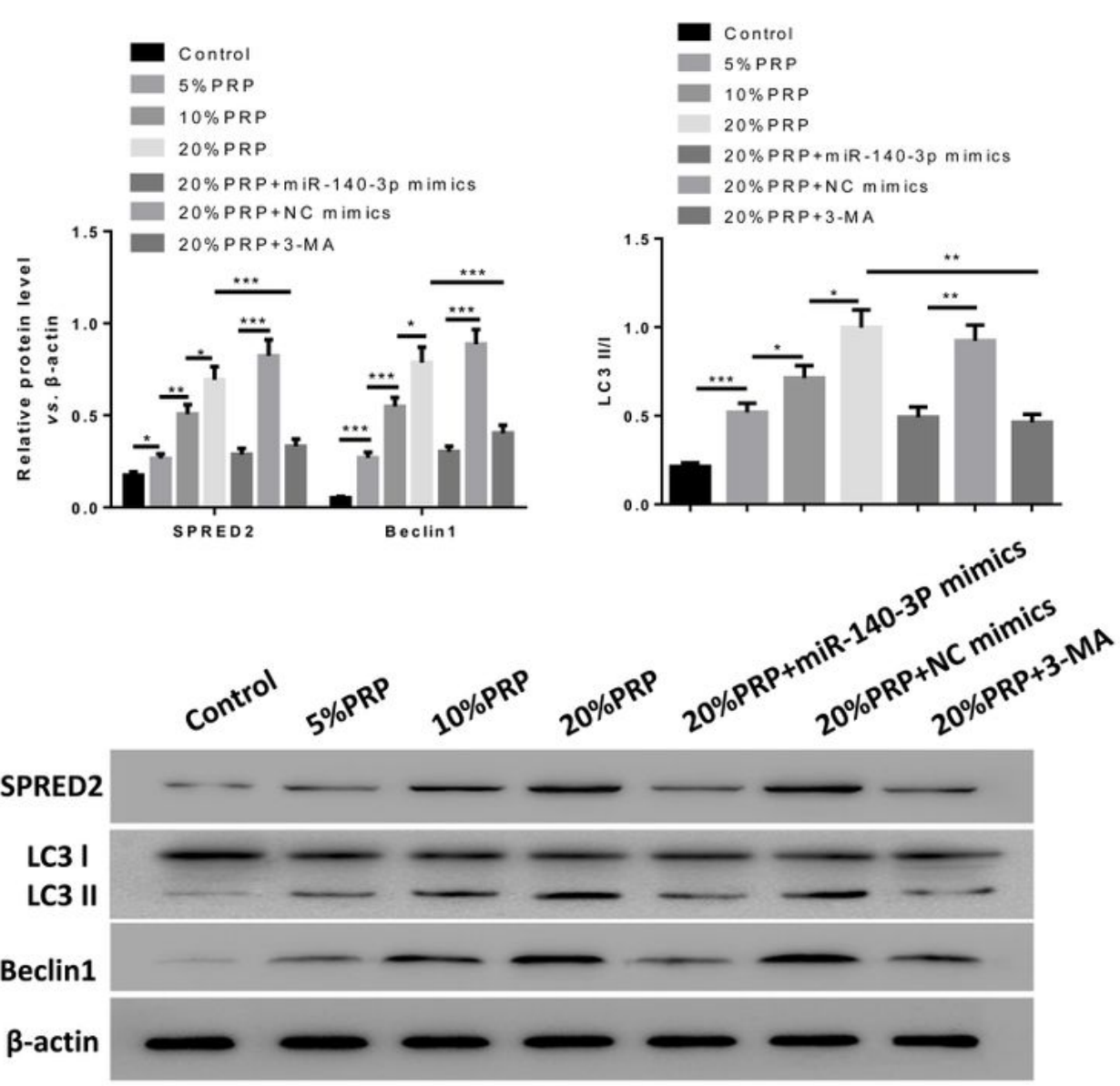

\section{Figure 2}

PRP activates autophagy and up-regulated SPRED2 expression of human BMSCs through suppressing miR-140-3p expression. (A) Expression of SPRED2 mRNA was detected by qRT-PCR. (B) Western blotting was used to evaluate the protein level of SPRED2, LC3 I, LC3II and Beclin1. ${ }^{\star} P<0.05,{ }^{\star *} P<0.01,{ }^{\star \star \star} P<0.001$. 
A

Position 1523-1530 of SPRED2 3'UTR

Has-miR-140-3p

B

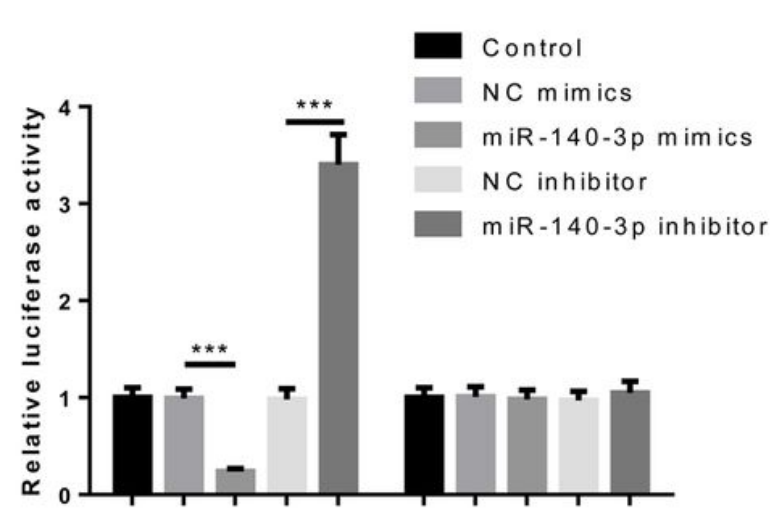

D

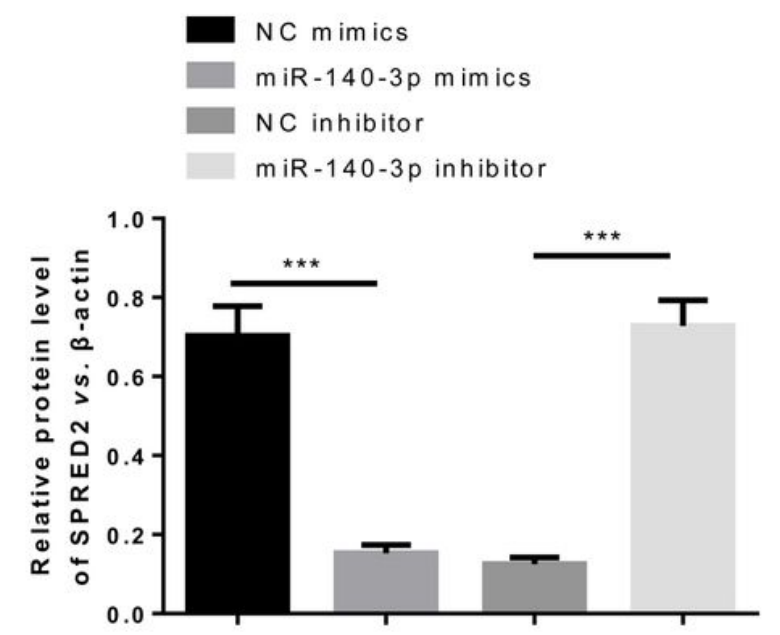

5'... CCCGCCACGAUCUUUCUGUGGUA...

3' GGCACCAAGAUGGGACACCAU
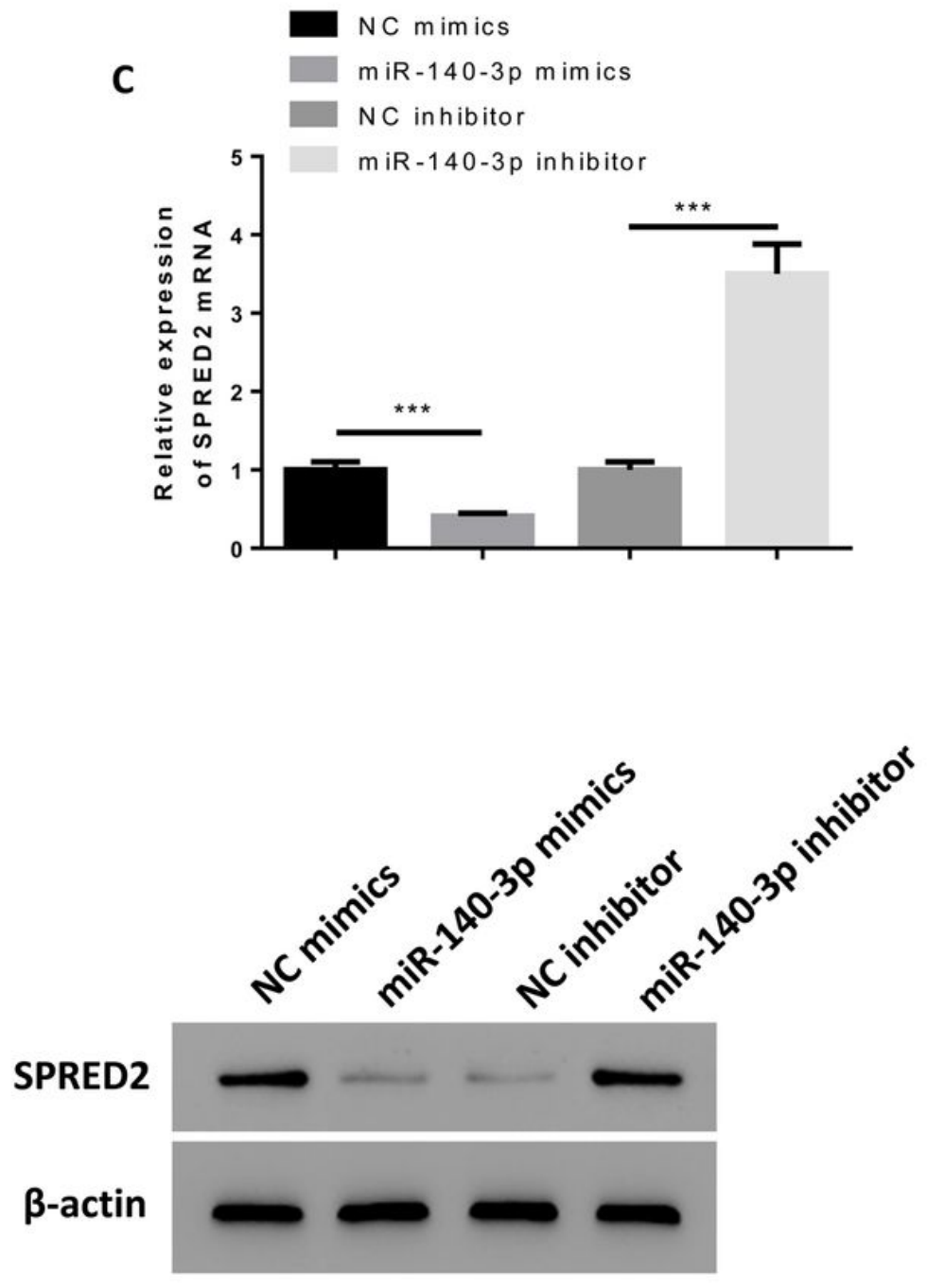

Figure 3

MiR-140-3p negatively regulated SPRED2 expression. (A) The predicted binding sites between miR-140-3p and SPRED2 are illustrated. (B) Cells were transfected with miR-140-3p mimics, miR-140-3p inhibitor or corresponding NCs, respectively. The targeting relationship between miR-140-3p and SPRED2 was identified by luciferase reporter assay. (C) Expression of SPRED2 was detected by qRT-PCR. (D) Protein expression was determined by western blotting. ${ }^{*} P<0.05,{ }^{* *} P<0.01,{ }^{* * *} P<0.001$. 
A

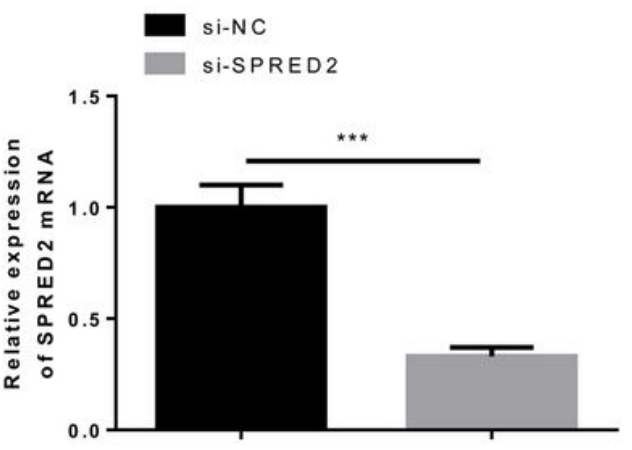

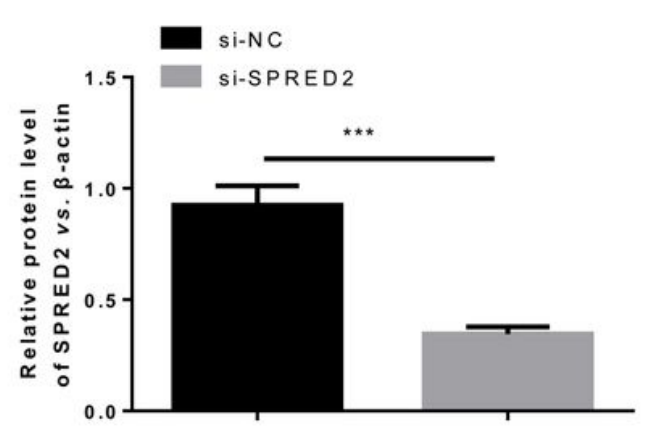

\section{B}

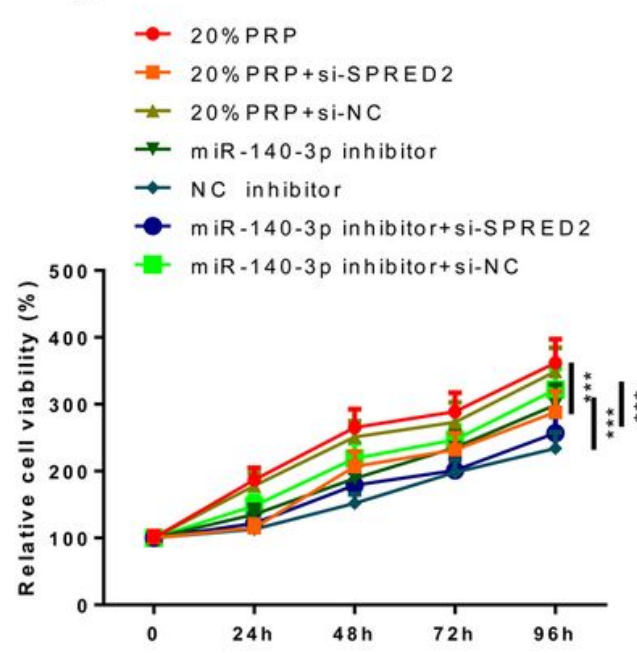

C

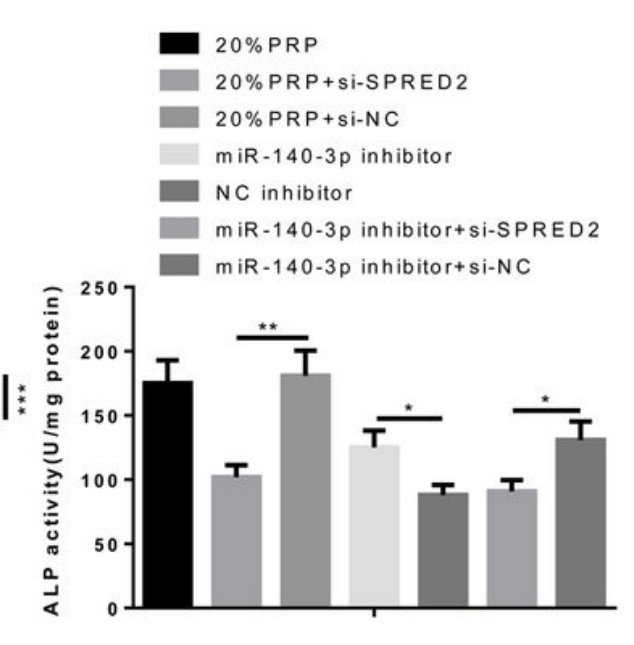

D

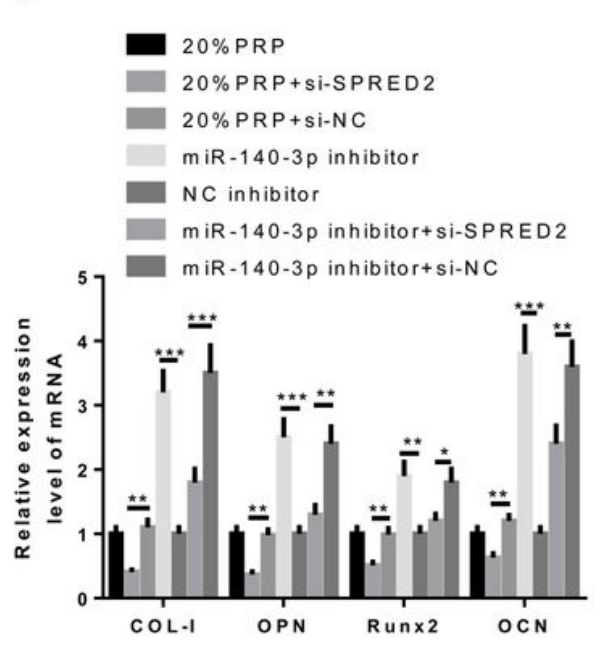

E

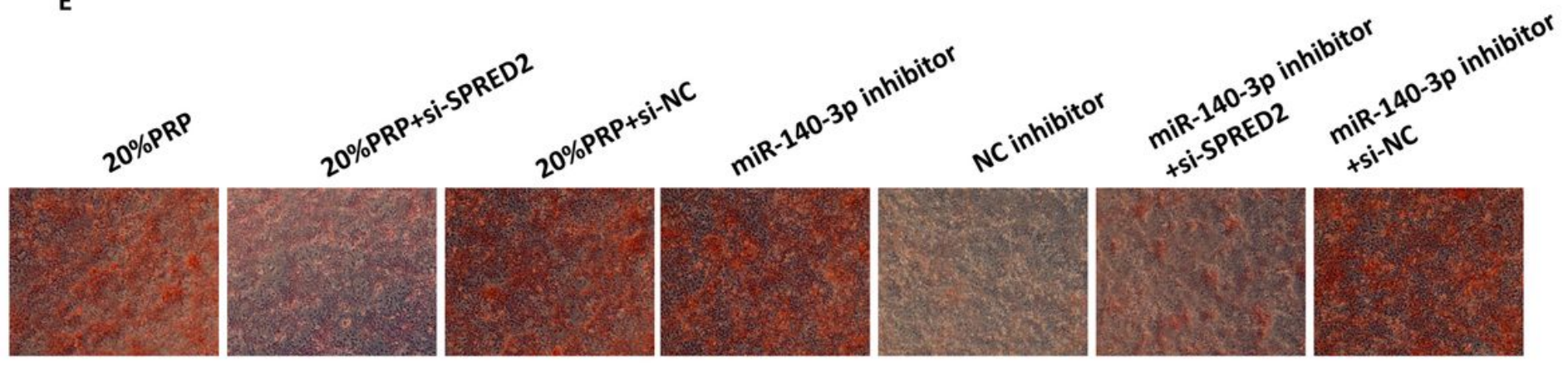

\section{Figure 4}

Inhibition of miR-140-3p enhances cell proliferation and osteogenic differentiation of BMSCs by negatively regulating SPRED2 expression. (A) Cells were transfected with si-SPRED2 or si-NC. The transfection efficiency was detected by qRT-PCR and western blotting. (B) Cell proliferation was determined by MTT assay in cells treated with $20 \%$ PRP, cells treated with $20 \%$ PRP and transfected with si-SPRED2 or si-NC, cells transfected with miR-140-3p inhibitor with/without si-SPRED2 and cells transfected with NCs. (C) ALP activity was evaluated. (D) Expression of COL-1, OPN, Runx2 and OCN was 
measured by qRT-PCR. (E) Calcium deposition was evaluated by Alizarin red staining assay. ${ }^{*}<<0.05$, $\star \star \mathrm{P}<0.01, * \star * \mathrm{P}<0.001$.

A

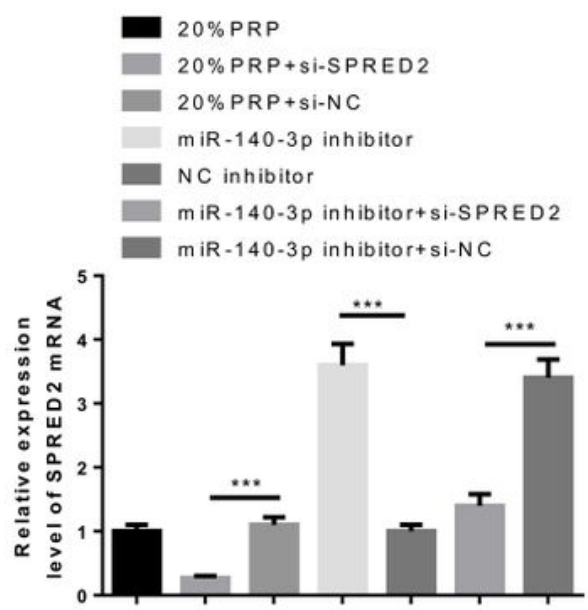

B
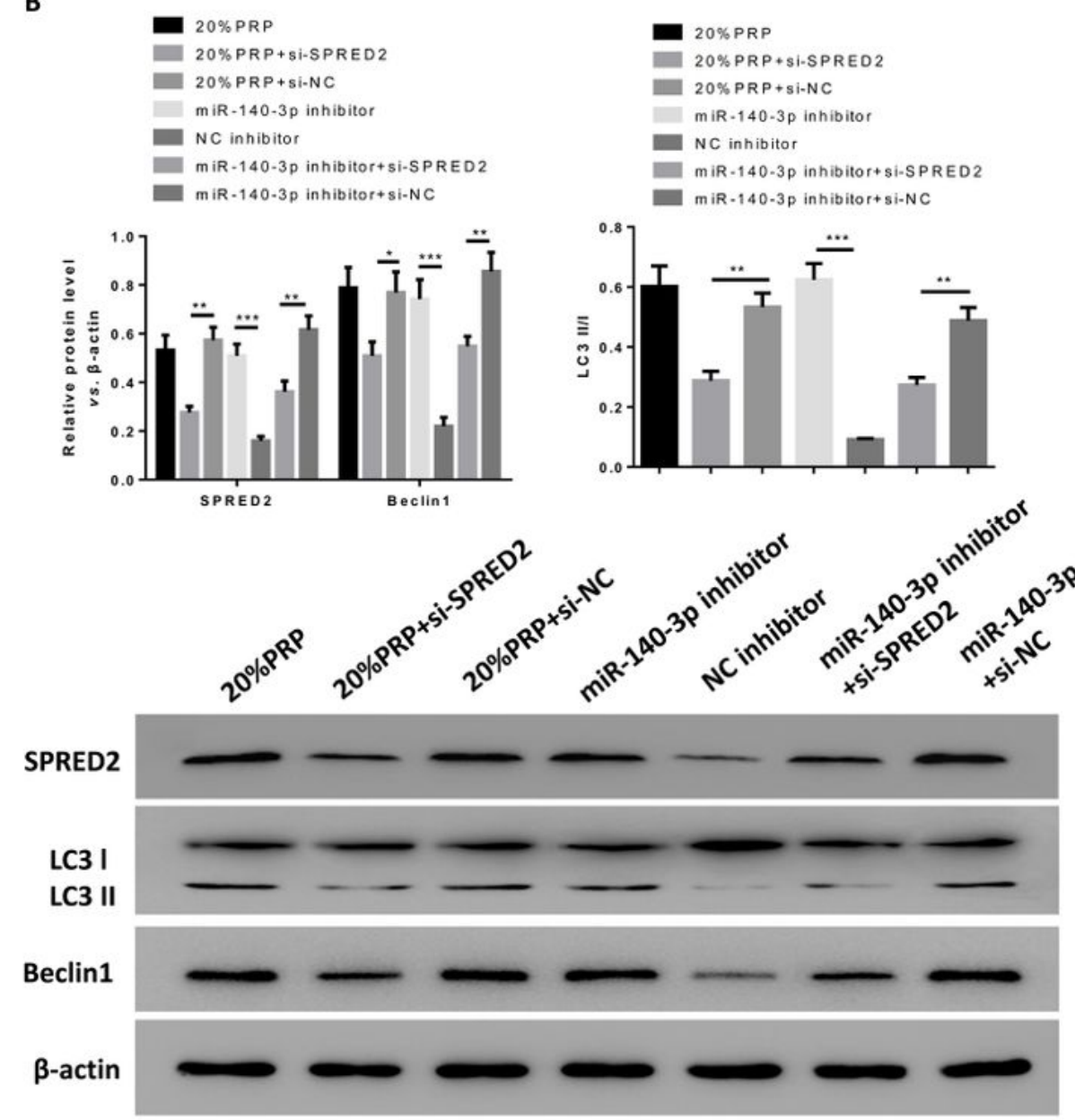

Figure 5

Knockdown of miR-140-3p enhances autophagy in human BMSCs through negatively regulating SPRED2 expression. (A) Expression of SPRED2 was detected by qRT-PCR. (B) Western blotting was used to evaluate the protein level of SPRED2, LC3 I, LC3II and Beclin1. ${ }^{*}<<0.05,{ }^{*} \mathrm{P}<0.01, * * * P<0.001$. 